\title{
Splenic contraction is enhanced by exercise at simulated high altitude
}

\author{
Angelica Lodin-Sundström ${ }^{1,3} \cdot$ Pontus Holmström $^{1}$ (1) $\cdot$ Marcus Ekstam $^{1} \cdot$ Daniel Söderberg $^{1} \cdot$ Erika Schagatay $^{1,2}$
}

Received: 2 October 2020 / Accepted: 9 February 2021 / Published online: 8 March 2021

(c) The Author(s) 2021

\begin{abstract}
Purpose Splenic contraction increases circulating hemoglobin $(\mathrm{Hb})$ with advantages during hypoxia. As both hypoxia and exercise have been shown to be important separate triggers of splenic contraction we aimed to investigate if the spleen response to simulated high altitude (HA) is enhanced by superimposing exercise.

Method Fourteen healthy volunteers (seven females) performed the following protocol in a normobaric environment sitting on an ergometer cycle: $20 \mathrm{~min}$ rest in normoxia; $20 \mathrm{~min}$ rest while breathing hypoxic gas simulating an altitude of $3500 \mathrm{~m}$; $10 \mathrm{~min}$ exercise at an individually set intensity while breathing the hypoxic gas; $20 \mathrm{~min}$ rest in hypoxia; and finally $20 \mathrm{~min}$ rest in normoxia. Spleen measurements were collected by ultrasonic imaging and venous Hb measured at the end of each intervention.

Result Mean \pm SD baseline spleen volume during normoxic rest was $280 \pm 107 \mathrm{~mL}$, the volume was reduced by $22 \%$ during rest in hypoxia to $217 \pm 92 \mathrm{~mL}(p<0.001)$ and by $33 \%$ during exercise in hypoxia $(189 \mathrm{~mL} ; p<0.001)$. Hb was $140.7 \pm 7.0 \mathrm{~g} / \mathrm{L}$ during normoxic rest and $141.3 \pm 7.4 \mathrm{~g} / \mathrm{L}$ during hypoxic rest (NS), but increased by $5.3 \%$ during hypoxic exercise $(148.6 \pm 6.3 \mathrm{~g} / \mathrm{L} ; p<0.001)$. Spleen volume and $\mathrm{Hb}$ were stepwise changed back to baseline at cessation of exercise and return to normoxia.

Conclusion Splenic contraction is induced by hypoxia and further enhanced by superimposing exercise, and reduced when exercise ceases, in a step-wise manner, showing that the tonic but partial contraction observed in long-term field expeditions to HA may occur also in the short term. This "graded response" may be beneficial during acclimatization to HA, to cope with moderate chronic hypoxia during rest while allowing additional enhancement of oxygen carrying capacity to overcome short bouts of extreme hypoxia caused by exercise.
\end{abstract}

Keywords Spleen size $\cdot$ Oxygen carrying capacity $\cdot$ Normobaric hypoxia $\cdot$ Hemoglobin $\cdot$ Performance

Abbreviations
$\begin{array}{ll}\text { ES } & \text { Effect size } \\ \text { HA } & \text { High altitude } \\ \mathrm{Hb} & \text { Hemoglobin concentration } \\ \mathrm{HR} & \text { Heart rate } \\ \text { IAT } & \text { Individual anaerobic threshold } \\ \mathrm{La} & \text { Blood lactate }\end{array}$

Communicated by Guido Ferretti.

Angelica Lodin-Sundström

Angelica.Lodin-Sundstrom@miun.se

1 Department of Health Sciences, Mid Sweden University, Östersund, Sweden

2 Swedish Winter Sports Research Centre, Östersund, Sweden

3 Environmental Physiology Group, Department of Nursing Science, Mid Sweden University, Holmgatan 10, 85170 Sundsvall, Sweden

$\begin{array}{ll}\mathrm{O}_{2} & \text { Oxygen } \\ \mathrm{PO}_{2} & \text { Partial pressure of oxygen } \\ \mathrm{RPE} & \text { Rating of perceived exertion } \\ \mathrm{SaO}_{2} & \text { Arterial oxygen saturation } \\ \mathrm{SpO}_{2} & \text { Peripheral oxygen saturation } \\ \mathrm{VC} & \text { Vital capacity } \\ \mathrm{VO}_{2 \max } & \text { Maximal oxygen uptake }\end{array}$

\section{Introduction}

Approximately 30-40 million non-acclimatized lowlanders travel to high altitude (HA) each year (Neupane and Swenson 2017). As a result, they are exposed to the decreasing atmospheric pressure and reduced partial pressure of oxygen $\left(\mathrm{PO}_{2}\right)$ with associated reductions in arterial oxygen saturation $\left(\mathrm{SaO}_{2}\right)$ that follow with increasing altitude (Grocott et al. 2009; West 2012), inevitably leading to progressive 
hypoxia with ascent. In response to the hypoxia acclimatization occurs, which eventually restores $\mathrm{O}_{2}$ homeostasis (West 2004). An early elevation in hemoglobin concentration ( $\mathrm{Hb}$ ) occurs, preceding the more long-term effect of acclimatization obtained after weeks by erythropoiesis (West 2012). It has been suggested that this early acclimatization is a result of dehydration associated with increased ventilation at HA, or to changes in plasma volume caused by sodium- and water-regulating hormonal responses to hypoxia (Bärtsch and Saltin 2008; Schmidt 2002). However, studies investigating spleen volume changes with erythrocyte release during hypoxia suggest that splenic contraction could be accountable for at least some of this effect (Schagatay et al. 2005; Richardson et al. 2008; Engan et al. 2014).

In many mammalian species, the spleen functions as an erythrocyte reservoir, recruited during strenuous activities that impose increased demands for $\mathrm{O}_{2}$ storage and transport (Barcroft and Stephens 1927; Guntheroth and Mullins 1963; Kramer and Luft 1951; Hurford et al. 1996). Red blood cells from the human splenic reservoir have been found to increase hematocrit (Hct) and $\mathrm{Hb}$ during exercise (Sandler et al. 1984; Hoka et al. 1989; Flamm et al. 1990; Laub et al. 1993), eupneic hypoxia (Richardson et al. 2008; LodinSundström and Schagatay 2010), and apneic diving (Hurford et al. 1990; Schagatay et al. 2001; Espersen et al. 2002; Bakovic et al. 2003). The increase in Hb across a series of maximal apneas was associated with prolonged apneic duration in intact participants, whereas splenectomized participants exhibited neither $\mathrm{Hb}$ elevation nor prolonged apneic duration (Schagatay et al. 2001).

Splenic contraction occurs also during exercise, resulting in release of stored erythrocytes into systemic circulation (Froelich et al. 1988; Laub et al. 1993). Stewart and associates (2003) found no change in spleen volume following 5, 10 , and 15 min of exercise at $60 \%$ maximal oxygen uptake $\left(\dot{\mathrm{V}}_{2 \text { max }}\right)$, but during maximal exercise, spleen volume was reduced by $56 \%$ and concluded that splenic contraction is an active response and may occur in an intensity-dependent manner with heavier exercise. Previous research found that the spleen contracts during $20 \mathrm{~min}$ of normobaric hypoxia $\left(\mathrm{F}_{\mathrm{i}} \mathrm{O}_{2} 12.8 \%\right.$ ), leading to an elevation of $\mathrm{Hb}$ by $2.1 \%$ (Richardson et al. 2008). In another study, comparing splenic contraction in two different hypoxic situations: apnea and 20 min rest during normobaric hypoxia $\left(\mathrm{F}_{\mathrm{i}} \mathrm{O}_{2} 14 \%\right)$, authors found that splenic contraction was twice as strong during apnea despite similar $\mathrm{SpO}_{2}$ levels (Lodin-Sundström and Schagatay 2010). However, all these studies suggested that the spleen may be important also during HA.

In a recent field expedition to Mount Everest base camp, baseline spleen volume at low altitude was found to be inversely associated with acute mountain sickness symptoms during ascent in non-acclimatized lowlanders (Holmström et al. 2019). This association indicates that individual spleen volume affects tolerance to HA hypoxia, likely by introducing stored erythrocytes into systemic circulation, thereby enhancing $\mathrm{O}_{2}$-carrying capacity. Spleen volume and contraction have also been found to be larger in elite climbers summiting Mount Everest compared to recreational trekkers (Schagatay et al. 2020a), but similar to that of successful freedivers who are regularly exposed to intermittent hypoxia (Schagatay et al. 2012). Holmström and colleagues (2020a) also found larger spleen volumes and contraction in Sherpa of highland origin compared to native Nepalese lowlanders, indicating a genetic component involved in spleen size development at HA. However, when the authors in the same study compared differences between Sherpa who live at HA and those who have migrated to low altitude, it was found that the Sherpa living at HA had larger spleen volume, which may indicate that human spleen size also is determined by environmental exposure to HA.

Baseline spleen volume has also been found to decrease in size during incremental ascent at HA (Schagatay et al. 2020b; Holmström et al. 2020b), attesting to a tonic splenic contraction during ascent, with a further volume reduction in response to exercise (Schagatay et al. 2020b) or apneainduced acute hypoxia (Holmström et al. 2020b). Collectively, these two field studies demonstrated a potentially important role of the splenic contraction at HA. Nevertheless, the combined effects of hypoxia and exercise on splenic contraction need further laboratory investigation to reveal if and to what extent the combined stimuli could induce a progressive, or graded response in the short term, which would seem functional at HA. Splenic contraction can develop fast and is abolished within 10 min (Schagatay et al. 2005), making it a potentially useful mechanism for rapid regulation of the circulating $\mathrm{Hb}$, optimizing the demands on $\mathrm{O}_{2}$ transport and those to keep viscosity at an acceptable level to limit cardiovascular stress. The present study explores this possibility by monitoring spleen volume during normoxia and when hypoxia and exercise are superimposed and then removed in a step-wise manner. We hypothesized that (a) rest in normobaric hypoxia would induce splenic contraction and (b) that the superimposed exercise would induce an enhanced splenic contraction resulting in further increase in $\mathrm{Hb}$, and that the response is reversed when these stimuli are subsequently removed.

\section{Methods}

Fourteen healthy volunteers [seven males and seven females: age; $25 \pm 3$ years; height $177 \pm 11 \mathrm{~cm}$; weight $76 \pm 14 \mathrm{~kg}$ and vital capacity (VC) $5.1 \pm 1.1 \mathrm{~L}]$ volunteered for the study. The participants were involved in physical training for $6.6 \pm 3.5 \mathrm{~h}$ per week but none was altitude-acclimatized. Participants were non-smokers but one regularly used snuff 
tobacco. They received written and oral information about the study and signed an informed consent form. The study complied with the Declaration of Helsinki and was approved by the local human ethics board.

\section{Procedures and measurements}

Each participant performed an individual anaerobic threshold test (IAT) on a cycle ergometer, to establish their individual exercise capacity (load) for the hypoxic test (details below). On a separate occasion after the IAT test, splenic function was measured at rest and during exercise in a simulated hypoxic environment (Hypoxic test). On both occasions participants arrived at the laboratory in a fasted and resting state, after at least $2 \mathrm{~h}$ without food or straining physical activity, and at least $1 \mathrm{~h}$ without having ingested any beverages.

\section{Individual anaerobic threshold test}

The participants performed a submaximal step-incremental exercise test in normoxia on a cycle ergometer to measure their IAT to establish individual exercise capacity for determination of the load in the hypoxic test. The IAT is defined as the metabolic rate where the elimination of blood lactate (La) during exercise is maximal and equal to the rate of diffusion of La into the blood (Stegmann et al. 1981). Consequently, power outputs above IAT will result in a progressive metabolic acidosis, and exercise time to exhaustion inversely related to the amount of exercise work rate exceeding IAT (Stegmann and Kindermann 1982). The IAT test was based on the maximal step-incremental exercise test by McLellan and Jacobs (1993) involving continuous exercise on a cycle ergometer (Monark ergomedic $828 \mathrm{E}$, Monark Exercise AB, Vansbro, Sweden). Starting at $30 \mathrm{~W}(\mathrm{~W})$, the intensity was increased in increments of $30 \mathrm{~W}$ every $4 \mathrm{~min}$. Thirty seconds before each incremental step, participants were asked to rate their perceived exertion (RPE) from 6 to 20 (Borg 1998). Heart rate (HR) was measured continuously (Polar RS 800, Polar Electro OY, Kempele, Finland), and capillary blood samples taken from the finger for lactate (La) analysis (Biosen 5140, EKF Diagnostic, Magdeburg, Germany). Participants pedaled with 60 revolutions per minute. The IAT test was terminated when $\mathrm{La}>4 \mathrm{mmol} / \mathrm{L}$ or when the participant rated 17 on the RPE scale. Even though the IAT, as described by McLellan and Jacobs (1993) and Stegmann and Kindermann (1982) entails maximal effort, these termination endpoints were included as precautionary measures to limit any health risks possibly associated with maximal exercise in hypoxia. That load was used for each individual in the hypoxic test. If the change of La between the final two steps differed by only $1 \mathrm{mmol} / \mathrm{L}$ or less, the participants load applied to the hypoxic test was set one step (30 W) below this load.

\section{Hypoxic exposure and exercise test}

Each participant had their anthropometric data and VC measured while standing (Vitalograph Compact 11, Vitalograph, Buckingham, England), with the larger of $3 \mathrm{VC}$ measurements used. An intravenous catheter (BD Venflon Pro, Becton Dickinson Infusion Therapy AB, Helsingborg, Sweden) was placed in the antecubital region to collect serial blood samples.

Participants rested for 20 min sitting on a cycle ergometer to achieve a homeostatic state in normoxia ('Baseline'). Hypoxia was induced by placing a non-rebreathing mask over the participants' nose and mouth allowing continuous breathing of 14\% oxygen in nitrogen for $20 \mathrm{~min}$ (Hypoxico, Hypoxico Inc., New York USA) simulating an altitude of $3500 \mathrm{~m}$ ('3500 m'). Thereafter, participants performed 10 min of cycling with the constant load previously determined in the IAT test at a frequency of $60 \mathrm{rev}^{*} \mathrm{~min}^{-1}$ while continuously breathing hypoxic air through the mask ('3500work'). The exercise was followed by $20 \mathrm{~min}$ rest on the cycle ergometer, with participants still breathing hypoxic air through the mask ('3500-recovery'). Finally the mask was removed and the participants continued to rest in normoxia for another $20 \mathrm{~min}$ in the same position ('Baselinerecovery'; Fig. 1).

\section{Measurements}

Spleen measurements were collected every minute throughout the experiment using ultrasonic imaging (Mindray DP-6600, Shenzhen Mindray Bio-Medical Electronics Co., Ltd., Shenzhen, China; Fig. 1). Peripheral oxygen saturation $\left(\mathrm{SpO}_{2}\right)$ and HR were continuously recorded using a pulsoxymeter (Biox 3700e, Ohmeda, Madison, WI, USA) with a probe on the index finger. The continuously registered parameters were stored via a multichannel data acquisition system (BioPac Systems Inc., Goleta, CA, USA).

Venous blood samples were collected for $\mathrm{Hb}$ measurements during the last minute of each of the five exposures (Fig. 1). The volume of blood removed per participant was less than $25 \mathrm{~mL}$ including waste, with approximately $10 \mathrm{~mL}$ of sterile isotonic $\mathrm{NaCl}$ solution infused for rinsing the catheter. In one participant capillary blood samples were collected due to blood clot in the catheter.

\section{Data analysis}

The triaxial measurements of splenic length $(L)$, width $(W)$, and thickness $(T)$ were used to calculate volume with the Pilström equation $\left[L \pi\left(W T-T^{2}\right) / 3\right]$ (Schagatay et al. 2005). 


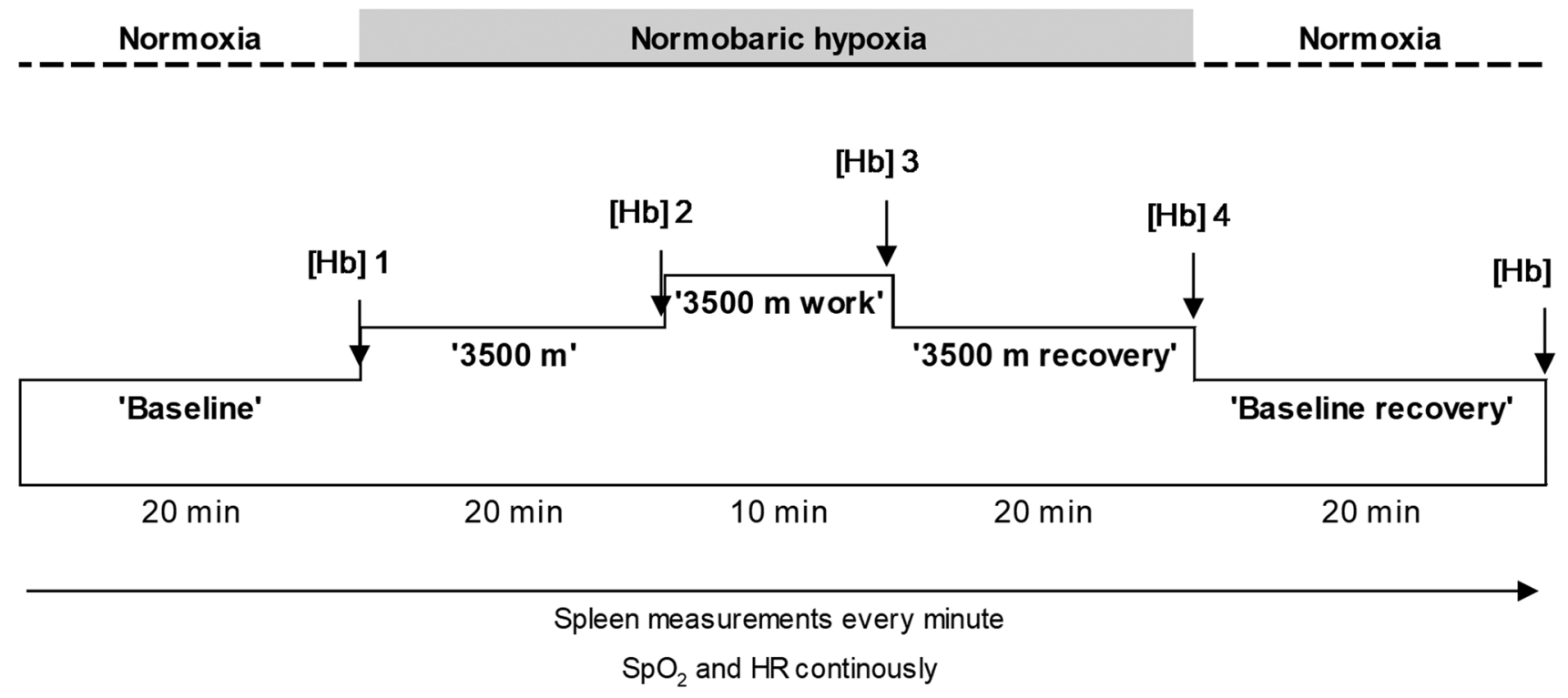

Fig. 1 Experimental procedure

Values from the final 2 min of each intervention were compared to baseline and among interventions.

Venous blood samples were analyzed in triplicate using an automated unit (Micros 60 Analyzer, ABX Diagnostics, Montpellier, France) and in cases where a deviation of triplicate samples of $>3 \mathrm{~g} / \mathrm{L}$ occurred, a fourth was analyzed. In one participant all blood samples were analyzed using a portable $\mathrm{Hb}$ analyzer (Hemocue $\mathrm{Hb} 201+$, Hemocue AB, Ängelholm, Sweden) due to capillary sampling.

\section{Statistical analysis}

Data are reported as mean \pm standard deviation (SD), unless otherwise stated. IBM SPSS statistics (version 25 for windows) was used to run appropriate statistical analysis. Normality distribution of data was tested using Kolmogorov-Smirnov and Shapiro-Wilk tests $(p>0.05)$. Assessments of interaction effects between the independent variables [treatment ('baseline; 3500 m', '3500-work'; '3500-recovery'; 'Baseline-recovery') and sex] on the dependent variables (spleen volume, $\mathrm{Hb}, \mathrm{SpO}_{2}$, and $\mathrm{HR}$ ) was conducted by a $2 \times 5$ two-way mixed ANOVA with Bonferroni corrections for repeated measures. Assessments of associations between dependent variables were conducted with bivariate correlation tests using Pearson product moment correlation coefficient $(r)$. Meaningfulness of treatment effects was estimated by the standardized mean difference [Cohen's $d$, effect size (ES)] computed as the mean difference divided by the pooled SD. ES was presented along with $95 \%$ confidence intervals ([CI]). An ES of $0.0-0.3$ was considered a small effect, $0.4-0.7$ medium and $>0.8$ a large effect (Lee 2016). Statistically significant differences were assumed at $p<0.05$.

\section{Results}

All participants successfully completed the experiment protocol. The two-way mixed ANOVA revealed a significant treatment effect on all dependent variables (spleen volume, $\mathrm{Hb}, \mathrm{SpO}_{2}$, and $\mathrm{HR}$ ). Nevertheless, there was no two-way interaction effect between males and females on the dependent variables during the experimental intervention (NS), indicating that these variables change equally between sexes in response to hypoxia and exercise. Therefore, all data for responses are presented with sexes pooled. Females had significantly smaller spleen volumes throughout the experiment ( $p=0.009)$ with a baseline volume of $218 \pm 73 \mathrm{~mL}$ compared to males at $342 \pm 103 \mathrm{~mL}(p=0.024 ; \mathrm{ES}=1.39$ [0.14-2.44]). There was no difference in $\mathrm{Hb}, \mathrm{HR}$ or $\mathrm{SpO}_{2}$ between females and males throughout the experiment (NS).

\section{Spleen response}

Baseline spleen volume in normoxic air was $280 \pm 107 \mathrm{~mL}$. Splenic contraction developed gradually during '3500 m' and had decreased to $217 \pm 92 \mathrm{~mL}$ (by $22 \%$ ) after $20 \mathrm{~min}$ (Fig. $2 ; p<0.001 ; \mathrm{ES}=0.63$ [ -0.15 to 1.37$]$ ). The contraction was enhanced after '3500-work', wherein spleen volume was reduced to $189 \pm 78 \mathrm{~mL}$ (by $33 \%$ ) after $10 \mathrm{~min}$ of exercise (Fig. 2; $p<0.001 ; \mathrm{ES}=0.97$ [0.16-1.72]). Spleen volume increased during '3500-recovery' to $244 \pm 108 \mathrm{~mL}$ (NS; ES $=0.33[-0.42$ to 1.07$]$ at $20 \mathrm{~min}$. Spleen volume 


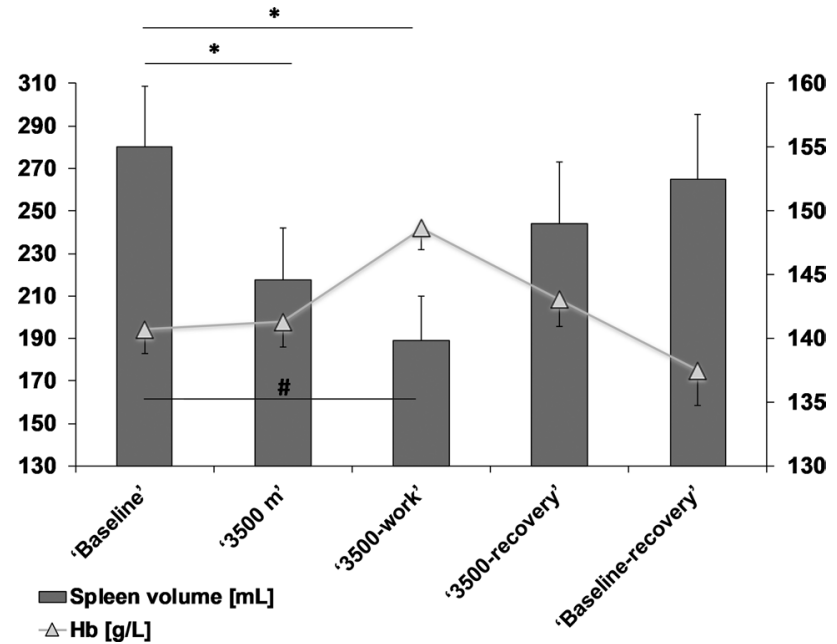

Fig. 2 Spleen volume and $\mathrm{Hb}$ changes during the experimental procedure. Standard error of the mean (SEM) is used to indicate the sample distribution. Significant difference at $p<0.001$ is indicated by $*$ for spleen volume and \# for $\mathrm{Hb}$

was further increased to $265 \pm 113 \mathrm{~mL}$ in normoxia during 'baseline-recovery', where it reached baseline values within 14 min (Fig. 2; NS; ES $=0.14$ [ -0.61 to 0.87$]$ ).

Baseline spleen volume was strongly associated with the magnitude of the contraction throughout the experiment: '3500 m' $(r=0.942, p<0.001)$; '3500-work' $(r=0.887$, $p<0.001)$; '3500-recovery' ( $r=0.932, p<0.001)$; 'Baseline-recovery' $(r=0.902, p<0.001)$.

\section{Hematological response}

Baseline $\mathrm{Hb}$ was $140.7 \pm 7.0 \mathrm{~g} / \mathrm{L}$ at the end of 'Baseline' normoxic rest, and after $20 \mathrm{~min}$ at ' $3500 \mathrm{~m}$ ' $\mathrm{Hb}$ was at $141.3 \pm 7.4 \mathrm{~g} / \mathrm{L}$ (Fig. 2; NS; ES $=0.00$ [ -0.74 to 0.74$]$ ). At the end of '3500-work', Hb had increased to $148.6 \pm 6.3 \mathrm{~g} / \mathrm{L}$, (by 5.3\%) from 'Baseline' (Fig. 2; $p<0.001$; $\mathrm{ES}=1.23$ [0.39-2.00]). By the end of '3500-recovery', Hb was $143.0 \pm 7.8 \mathrm{~g} / \mathrm{L}(\mathrm{NS} ; \mathrm{ES}=0.27$ [0.49-1.00]) and it was restored by the end of 'Baseline-recovery' at $137.5 \pm 10$ (NS; $\mathrm{ES}=0.35[-0.41$ to 1.08$])$.

\section{Cardiovascular parameters}

Baseline $\mathrm{SpO}_{2}$ and $\mathrm{HR}$ were $97 \pm 1 \%$ and $78 \pm 9 \mathrm{bpm}$, respectively. After $20 \mathrm{~min}$ at ' $3500 \mathrm{~m}$ ', $\mathrm{SpO}_{2}$ had decreased to $88 \pm 4 \%$ (by $9 \% ; p<0.001 ; \mathrm{ES}=3.09$ [1.92-4.08]) and HR had increased to $82 \pm 10 \mathrm{bpm}$ (by $5 \%$; NS; ES $=0.42$ [0.34-1.16]). By the end of exercise $\mathrm{SpO}_{2}$ had decreased to $77 \pm 6 \%$ (by $13 \%$ from ' 3500 m'; $p<0.001$; $\mathrm{ES}=2.16$ [1.17-3.02]) and HR increased to $125 \pm 29$ bpm (by $52 \%$ from ' $3500 \mathrm{~m}$ '; $p<0.001$; $\mathrm{ES}=1.98$ [1.03-2.82]). By the end of the 20 min recovery at sea-level 'Baseline-recovery', all parameters had returned to pre-exposure baseline values $\left(\mathrm{SpO}_{2}: 97 \pm 1 \%\right.$; HR: $79 \pm 11$ bpm; NS).

\section{Discussion}

We investigated the combined effect of hypoxia and exercise on splenic contraction during simulated HA equivalent to $3500 \mathrm{~m}\left(\mathrm{~F}_{\mathrm{i}} \mathrm{O}_{2} 14 \%\right)$ in non-acclimatized lowlanders. The principal findings were that during rest, normobaric hypoxia induced a partial splenic contraction, which was enhanced with superimposed exercise and subsequently reduced again with return to rest in hypoxia, and further reduced by return to normoxic air. The spleen volume decreases during hypoxia and exercise was accompanied by Hb elevation, and the return to baseline spleen size with a reduction in $\mathrm{Hb}$. Thus, the spleen responded in a step-wise manner to the progressively increased and decreased stimuli, which demonstrates that the spleen has the ability to fine tune the amount of circulating red cells in the short term to optimize between metabolic needs and cardiovascular load (sheer stress). This is the first laboratory study confirming earlier long-term field observations suggesting that, when non-acclimatized lowlanders are exposed to HA, a partial tonic splenic contraction is induced on which additional stimuli may induce yet a more powerful response (Richardson and Schagatay 2007; Holmström et al. 2020b; Schagatay et al. 2020b). The present study shows such a fine-tuned graded response may also be induced in the short term.

Richardson and Schagatay (2007) found an attenuated apnea-induced $\mathrm{Hb}$ increase during ascent to $5100 \mathrm{~m}$, whereby they concluded that hypobaric hypoxia at HA causes a tonic splenic contraction that most likely leads to elimination of further apnea-induced $\mathrm{Hb}$ increases. Holmström and colleagues (2020b) measured spleen volume and $\mathrm{Hb}$ during incremental ascent at $\mathrm{HA}$ in non-acclimatized lowlanders. They reported that baseline spleen volume decreased by approximately $14 \%$ per $1000 \mathrm{~m}$ ascent, which was also negatively associated with baseline $\mathrm{Hb}$, further attesting to a tonic splenic contraction during ascent at HA. However, in addition to supporting the existence of a tonic splenic contraction in non-acclimatized lowlanders during HA exposure, with a putative effect to enhance oxygen uptake, our findings contributes new knowledge of a short-term splenic ability to transiently fine-tune circulating erythrocytes in an acute phase, likely to optimize between increased demands on $\mathrm{O}_{2}$ transportation to muscles and hypoxia sensitive organs during bouts of exercise and reducing viscosity during rest to reduce cardiovascular stress. While the level of hypoxia is similar, it should be noted that normobaric hypoxia is different from hypobaric hypoxia, which may result in differences in hypoxia-induced 
responses of some variables e.g., ventilation, $\mathrm{NO}$, and fluid retention (Coppel et al. 2015).

Our data displayed a significant splenic contraction at rest in hypoxia, which is in line with previous research that suggests that hypoxia is an important trigger of the splenic contraction (Richardson et al. 2008; Lodin-Sundström and Schagatay 2010). The magnitude of $22 \%$ reduction in spleen volume observed in the present investigation during rest at simulated altitude of $3500 \mathrm{~m}$, was in the range of responses previously reported, i.e., a decrease in spleen volume by $18 \%$ after resting participants were exposed to normobaric hypoxia corresponding to an altitude of $4100 \mathrm{~m}$, which also resulted in a Hb elevation of $2.1 \%$ (Richardson et al. 2008). Lodin-Sundström and Schagatay (2010) reported a splenic contraction by $16 \%$ during rest in normobaric hypoxia, corresponding to an altitude of $3300 \mathrm{~m}$. Since resting spleen volume and contraction vary between individuals (Prassopoulos et al. 1997), the different reported magnitudes of spleen contraction may be due to individual differences between subjects in the relatively small groups studied.

While splenic contraction occurs during exercise (Laub et al. 1993; Stewart et al. 2003), apnea (Schagatay et al. 2005), normobaric hypoxia (Richardson et al. 2008; LodinSundström and Schagatay 2010) hypobaric hypoxia (Holmström et al. 2020b; Schagatay et al. 2020b) and during handgrip exercise (Frances et al. 2008), the mechanisms behind human splenic contraction have not been fully clarified. Nevertheless, hypoxia appears to be a major factor inducing the response (Richardson et al. 2008; Lodin-Sundström and Schagatay 2010). The splenic nerve is almost entirely (98\%) composed by sympathetic nerve fibers and may be affected by increased hypoxia-induced sympathetic output. More rapid changes in spleen volume have also been reported in apneic situations, with immediate onset of apnea before hypoxia has developed, which has been addressed to withdrawal of inhibitory baroreceptor activity and reduced cardiac output, resulting in a significant fall in mean arterial pressure increasing the sympathetic output (Bakovic et al. 2003; Palada et al. 2007). Thus, both rapid neural and progressive chemoreceptor input may elicit spleen contraction, with the latter being a likely candidate in this study. The severe exercise-induced $\mathrm{O}_{2}$ desaturation indicates systemic hypoxemia, conceivably the chemoreceptors may regulate the response, where $\mathrm{PO}_{2}$ below a certain level may likely cause a more pronounced splenic contraction if the hypoxemic stress is severe enough. With rest in mild hypoxia the effects are minor, but with onset of exercise an additional neural input is likely involved.

The enhanced splenic contraction with exercise reflects the ability of the spleen to continuously regulate circulating $\mathrm{Hb}$ to the varying demands on the $\mathrm{O}_{2}$ transportation system associated with different levels of exertion and consequent muscle $\mathrm{O}_{2}$ demands. This fine-tuned regulation may allow sustained performance at HA even though the physiological demands increase with exercise. Dane et al. (2006) observed that the spleen blood serves an important function for $\mathrm{O}_{2}$ transportation during exercise in mammals with high-aerobic capacity like dogs, and showed that the elevated $\mathrm{Hb}$ due to splenic contraction contributed to an increased diffusion of $\mathrm{O}_{2}$ from the lung to the blood and from blood to tissue. This suggests that the spleen is an important reservoir also during exercise in situations when the body is already under stress, and shows that the spleen was only partially contracted during $3500 \mathrm{~m}$ altitude simulation.

Maximum aerobic performance is reduced in hypoxia (Bärtsch and Saltin 2008), hence exercise in hypoxia entails working at a higher relative workload compared to normoxia. Therefore, even light work will impose a substantial stress to the system. It is, therefore, difficult to distinguish between the effect of enhanced hypoxia in itself and those imposed by the workload. It has previously been suggested that splenic contraction develops in an intensity-dependent manner during exercise (Stewart et al. 2003). This is the first study to demonstrate this intensity-dependent contraction as a graded response to hypoxia as well.

Regardless of input, the reversible nature of the response shows that the temporary enhancement of circulating $\mathrm{Hb}$ is not likely due to hemoconcentration as a result of extravasation of plasma, which is in accord with the previous studies (Schagatay et al. 2001; Bakovic et al. 2003). Hb concentrations may increase during exercise, suggested to be primarily due to rapid plasma water reductions, resulting from a transient fluid shift from intravascular to extravascular compartments (Jacobs et al. 2012). Nonetheless, elevated levels of erythrocytes by splenic contraction have also previously been suggested to contribute to the $\mathrm{Hb}$ concentration increase during exercise at sea level (Jacobs et al. 2012; Laub et al. 1993; Stewart et al. 2003), resulting in a 4-6\% elevation in Hct (Laub et al. 1993; Stewart et al. 2003) and $\mathrm{O}_{2}$-carrying capacity improvements by approximately 10\% (Schmidt and Prommer 2010). This is consistent with our findings of a reversible $\mathrm{Hb}$ increase during exercise in hypoxia by $5.3 \%$ and we, therefore, suggest that the splenic contraction that occurs during exercise in normobaric hypoxia contributes substantially to the $\mathrm{Hb}$ increase.

\section{Methodological considerations}

One limitation is that the IAT test was performed in normoxia for the test conducted in hypoxia. However, since all subjects served as their own control, this is not expected to have influenced the conclusions. The current study observed a marginal not significant $\mathrm{Hb}$ elevation when entering hypoxia compared to normoxia at rest. This was unexpected as previous research has displayed a $2.1 \% \mathrm{Hb}$ elevation resulting from splenic contraction in normobaric hypoxia 
equivalent to $4100 \mathrm{~m}\left(\mathrm{~F}_{\mathrm{i}} \mathrm{O}_{2} 12.8 \%\right)$ ascribed to hypoxiainduced splenic contraction (Richardson et al. 2008) but as our simulated altitude of $3500 \mathrm{~m}$ was lower, this could have resulted in a too small drop in $\mathrm{SpO}_{2}$. In addition, in a previous study, baseline spleen contraction and $\mathrm{Hb}$ elevation during rest was small and the main effect was seen with exercise (Schagatay et al 2020a, b). Another possible cause of the minor $\mathrm{Hb}$ effect, despite a significant spleen contraction is that we used the same time point for spleen measurements and blood samples, but there could be a circulatory delay of the Hb effect. The small sample size also did not allow a reliable conclusion to be done from the correlation analysis. The fact that previously reported effects of normobaric hypoxia at rest on $\mathrm{Hb}$ were also small and individual differences in $\mathrm{Hb}$ are large means that changes may possibly be undetected in a small sample, although we found significant stepwise splenic volume changes.

\section{Conclusions}

We found that the splenic contraction induced by hypoxia during rest is enhanced when exercise is superimposed, with a concomitant increase in circulating $\mathrm{Hb}$. The enhanced response with exercise is likely a result of the further decrease in $\mathrm{SpO}_{2}$. With normoxia both responses are fully recovered to baseline within $20 \mathrm{~min}$. This step-wise response suggests that the spleen may provide a means for a rapid and fine-tuned control of circulating erythrocytes with exercise at altitude, and a rapid reversal of this effect at exercise termination, limiting the period with increased viscosity. This clearly confirms results from earlier studies done in the field at varying environmental conditions, and shows these changes also occur in the short term. Together with earlier field studies this report supports the view that the moderate tonic splenic contraction with hypoxia at rest may contribute to coping with $\mathrm{HA}$ before any effects on $\mathrm{Hb}$ of erythropoiesis has developed, likely resulting in an increased hypoxia tolerance at HA. The remaining capacity for splenic contraction is recruited only during the transient excessive stress imposed by e.g., exercise.

Acknowledgements We like to express our gratitude to the participants and to Caroline Eriksson, Felix Schagatay, Helene Larsdotter Vigetun for assistance in the laboratory, to Glenn Björklund for advice on the protocol and to Alex Patrician for feedback on the manuscript.

Author contributions LSA: Idea and development of the research question and methods, pilot study, planning, analysis, article writing, and proof reading. HP: Development of the statistical analysis, article writing, and proof reading. EM: Methods development, pilot study, planning and organizing the laboratory, data collection, analysis, and proof reading. SD: Methods development, pilot study, planning and organizing the laboratory, data collection, analysis, and proof reading. SE: Development of the research question and methods, pilot study, planning and organizing the laboratory, data collection, analysis, article writing, and proof reading.

Funding Open access funding provided by Mid Sweden University. The study was financed by the Swedish National Centre for Research in Sports (CIF).

\section{Compliance with ethical standards}

Conflict of interest No competing financial interest exists.

Ethics approval The study was approved by the Regional Committee for Medical and Health Research Ethics in Umeå, Sweden and conducted in accordance with ethical standards of the 1964 Helsinki Declaration. All participants provided written informed consent before participation.

Open Access This article is licensed under a Creative Commons Attribution 4.0 International License, which permits use, sharing, adaptation, distribution and reproduction in any medium or format, as long as you give appropriate credit to the original author(s) and the source, provide a link to the Creative Commons licence, and indicate if changes were made. The images or other third party material in this article are included in the article's Creative Commons licence, unless indicated otherwise in a credit line to the material. If material is not included in the article's Creative Commons licence and your intended use is not permitted by statutory regulation or exceeds the permitted use, you will need to obtain permission directly from the copyright holder. To view a copy of this licence, visit http://creativecommons.org/licenses/by/4.0/.

\section{References}

Bakovic D, Valic Z, Eterovic D, Vukovic I, Obad A, Marinovic-Terzic I, Dujic Z (2003) Spleen volume and blood flow response to repeated breath-hold apneas. J Appl Physiol 95(4):1460-1466

Barcroft J, Stephens J (1927) Observations upon the size of the spleen. J Physiol 64(1):1-22

Bärtsch P, Saltin B (2008) General introduction to altitude adaptation and mountain sickness. Scand J Med Sci Sports 18(s1):1-10

Borg G (1998) Borg's perceived exertion and pain scales. Human Kinetics, Champaign

Coppel J, Hennis P, Gilbert-Kawai E, Grocott MP (2015) The physiological effects of hypobaric hypoxia versus normobaric hypoxia: a systematic review of crossover trials. Extreme Physiol Med 4(1):2

Dane DM, Hsia CC, Wu EY, Hogg RT, Hogg DC, Estrera AS, Johnson RL Jr (2006) Splenectomy impairs diffusive oxygen transport in the lung of dogs. J Appl Physiol 101(1):289-297

Engan H, Lodin-Sundström A, Schagatay F, Schagatay E (2014) The effect of climbing Mount Everest on spleen contraction and increase in hemoglobin concentration during breath holding and exercise. High Altitude Med Biol 15(1):52-57

Espersen K, Frandsen H, Lorentzen T, Kanstrup I-L, Christensen NJ (2002) The human spleen as an erythrocyte reservoir in divingrelated interventions. J Appl Physiol 92(5):2071-2079

Flamm SD, Taki J, Moore R, Lewis S, Keech F, Maltais F et al (1990) Redistribution of regional and organ blood volume and effect on cardiac function in relation to upright exercise intensity in healthy human subjects. Circulation 81(5):1550-1559

Frances MF, Dujic Z, Shoemaker JK (2008) Splenic constriction during isometric handgrip exercise in humans. Appl Physiol Nutr Metab 33(5):990-996 
Froelich J, Strauss H, Moore R, McKusick K (1988) Redistribution of visceral blood volume in upright exercise in healthy volunteers. J Nucl Med 29(10):1714-1718

Grocott MP, Martin DS, Levett DZ, McMorrow R, Windsor J, Montgomery HE (2009) Arterial blood gases and oxygen content in climbers on Mount Everest. N Engl J Med 360(2):140-149

Guntheroth WG, Mullins GL (1963) Liver and spleen as venous reservoirs. Am J Physiol Legacy Content 204(1):35-41

Hoka S, Bosnjak ZJ, Arimura H, Kampine JP (1989) Regional venous outflow, blood volume, and sympathetic nerve activity during severe hypoxia. Am J Physiol Heart Circ Physiol 256(1):H162-H170

Holmström P, Mulder E, Lodin-Sundström A, Limbu P, Schagatay E (2019) The magnitude of diving bradycardia during apnea at lowaltitude reveals tolerance to high altitude hypoxia. Front Physiol 10:1075

Holmström P, Bird J, Thrall S, Kalker A, Herrington B, Soriano J et al (2020a) The effects of incremental ascent to altitude on the diving response and splenic contraction during voluntary breath holding. J Exp Physiol. https://doi.org/10.1113/EP088571

Holmström P, Mulder E, Starfelt V, Lodin-Sundström A, Schagatay E (2020b) Spleen size and function in sherpa living high, sherpa living low and nepalese lowlanders. Front Physiol 11:647

Hurford WE, Hong S, Park YS, Ahn D, Shiraki K, Mohri M, Zapol WM (1990) Splenic contraction during breath-hold diving in the Korean ama. J Appl Physiol 69(3):932-936

Hurford WE, Hochachka PW, Schneider RC, Guyton GP, Stanek KS, Zapol DG et al (1996) Splenic contraction, catecholamine release, and blood volume redistribution during diving in the Weddell seal. J Appl Physiol 80(1):298-306

Jacobs RA, Lundby C, Robach P, Gassmann M (2012) Red blood cell volume and the capacity for exercise at moderate to high altitude. Sports Med 42(8):643-663

Kramer K, Luft UC (1951) Mobilization of red cells and oxygen from the spleen in severe hypoxia. Am J Physiol Legacy Content 165(1):215-228

Laub M, Hvid-Jacobsen K, Hovind P, Kanstrup I, Christensen NJ, Nielsen SL (1993) Spleen emptying and venous hematocrit in humans during exercise. J Appl Physiol 74(3):1024-1026

Lee DK (2016) Alternatives to P value: confidence interval and effect size. Korean J Anesthesiol 69(6):555

Lodin-Sundström A, Schagatay E (2010) Spleen contraction during 20 min normobaric hypoxia and $2 \mathrm{~min}$ apnea in humans. Aviat Space Environ Med 81(6):545-549

McLellan TM, Jacobs I (1993) Reliability, reproducibility and validity of the individual anaerobic threshold. Eur J Appl Physiol 67(2):125-131

Neupane M, Swenson ER (2017) High-altitude pulmonary vascular diseases. Adv Pulmonary Hypertension 15(3):149-157

Palada I, Obad A, Bakovic D, Valic Z, Ivancev V, Dujic Z (2007) Cerebral and peripheral hemodynamics and oxygenation during maximal dry breath-holds. Respir Physiol Neurobiol 157(2-3):374-381

Prassopoulos P, Daskalogiannaki M, Raissaki M, Hatjidakis A, Gourtsoyiannis N (1997) Determination of normal splenic volume on computed tomography in relation to age, gender and body habitus. Eur Radiol 7(2):246-248

Richardson M, Schagatay E (2007) Altitude attenuates apnea-induced increase in haemoglobin concentration. Environm Ergon XI I:90

Richardson M, Lodin A, Reimers J, Schagatay E (2008) Short-term effects of normobaric hypoxia on the human spleen. Eur J Appl Physiol 104(2):395-399

Sandler MP, Kronenberg MW, Forman MB, Wolfe OH, Clanton JA, Partain CL (1984) Dynamic fluctuations in blood and spleen radioactivity: splenic contraction and relation to clinical radionuclide volume calculations. J Am Coll Cardiol 3(5):1205-1211

Schagatay E, Andersson J, Hallén M, Pålsson B (2001) Selected contribution: role of spleen emptying in prolonging apneas in humans. J Appl Physiol 90(4):1623-1629

Schagatay E, Haughey H, Reimers J (2005) Speed of spleen volume changes evoked by serial apneas. Eur J Appl Physiol 93(4):447-452

Schagatay E, Richardson M, Lodin-Sundström A (2012) Size matters: spleen and lung volumes predict performance in human apneic divers. Front Physiol 3:173

Schagatay E, Holmström P, Mulder E, Limbu P, Schagatay FS, Engan H, Lodin-Sundström A (2020a) Spleen volume and contraction during apnea in Mt. Everest climbers and Everest base camp trekkers. High Altitude Med Biol 21(1):84-91

Schagatay E, Lunde A, Nilsson S, Palm O, Lodin-Sundström A (2020b) Spleen contraction elevates hemoglobin concentration at high altitude during rest and exercise. Eur J Appl Physiol. https://doi. org/10.1007/s00421-020-04471-w

Schmidt W (2002) Effects of intermittent exposure to high altitude on blood volume and erythropoietic activity. High Altitude Med Biol 3(2):167-176

Schmidt W, Prommer N (2010) Impact of alterations in total hemoglobin mass on V' O2max. Exerc Sport Sci Rev 38(2):68-75

Stegmann H, Kindermann W (1982) Comparison of prolonged exercise tests at the individual anaerobic threshold and the fixed anaerobic threshold of $4 \mathrm{mmol} \cdot \mathrm{L}-1$ lactate. Int J Sports Med 3(02):105-110

Stegmann H, Kindermann W, Schnabel A (1981) Lactate kinetics and individual anaerobic threshold. Int J Sports Med 2(03):160-165

Stewart IB, Warburton DE, Hodges AN, Lyster DM, McKenzie DC (2003) Cardiovascular and splenic responses to exercise in humans. J Appl Physiol 94(4):1619-1626

West JB (2004) The physiologic basis of high-altitude diseases. Ann Intern Med 141(10):789-800

West JB (2012) High-altitude medicine. Am J Respir Crit Care Med 186(12):1229-1237

Publisher's Note Springer Nature remains neutral with regard to jurisdictional claims in published maps and institutional affiliations. 